Fecha de recepción: octubre 2019 Fecha de aceptación: diciembre 2019 Versión final: diciembre 2021

\section{La identidad de los equipos de fútbol mexicanos a través de sus identificadores gráficos y su influencia en la cultura mexicana}

Mariana Noemí Campos Barragán ${ }^{1}$, Cynthia Lizette Hurtado Espinosa ${ }^{2}$ y

Miguel Angel Casillas López ${ }^{3}$

Resumen: En México, el fútbol se convierte en una pasión para muchos aficionados, y la cultura entorno a este deporte se representa con la expresión de las porras, asistencia a los estadios y reuniones sociales con los familiares y amigos. Son los identificadores gráficos de los equipos y su identidad en las playeras lo que proyecta la esencia de cada equipo, provocando una fuerte identificación de los aficionados con su equipo fomentando un sentido de pertenencia intenso hacia ellos. Este es un fenómeno que pocas empresas pueden lograr tan fehaciente en la mente del consumidor e incluso llegar a despertar un amor indescriptible. En este texto se documenta la evolución de los identificadores gráficos de los cinco equipos más representativos del fútbol mexicano de acuerdo a la revista Forbes, México 2018 que incluyen al Guadalajara, el Monterrey, el América, Santos Laguna, Tijuana, y algunos de los efectos que producen en sus seguidores.

Palabras clave: Identidad -Identificadores gráficos - fútbol - Cultura mexicana - Sentido de pertenencia.

[Resúmenes en inglés y portugués en las páginas 45-46]

(1) Mariana Noemí Campos Barragán: Profesor de asignatura de la Universidad de Guadalajara con licenciatura en Diseño para la comunicación gráfica y maestría en Metodología de la Enseñanza. Colaborador CA 790 enfocado a los procesos de comunicación y educación superior. Ha participado en actividades académicas y de gestión en el centro universitario de Arte, Arquitectura y Diseño. Mail: m_noemi17@hotmail.com

(2) Cynthia Lizette Hurtado Espinosa: Profesor investigador de la Universidad de Guadalajara con licenciatura en Diseño para la comunicación gráfica y maestría y doctorado en Metodología de la Enseñanza. Editora de la revista Zincografía, responsable del CA 790 enfocado a los procesos de comunicación y educación superior. Ha sido Coordinadora de investigación y posgrado y de la licenciatura en Diseño para la Comunicación Gráfica. Mail: cynthia.hurtado@academicos.udg.mx

(3) Miguel Angel Casillas López: Profesor de la Universidad de Guadalajara, entre sus líneas de investigación se encuentra Tecnología en el proceso de enseñanza aprendizaje. Es 
miembro del cuerpo académico UDG-CA-790 Procesos de Comunicación y Educación Superior. Ha sido coordinador de Tecnologías para el Aprendizaje, participando en actividades académicas y de gestión en la red universitaria. Mail: miguel.casillas@cuaad.udg.mx

\section{Introducción}

Cuando se habla de un deporte tan fuertemente arraigado como el fútbol en México, se genera una especie de culto alrededor de él, una pasión e intensidad que solamente quienes lo han vivido lo comprenden y sienten al equipo como suyo.

Para elegir los 5 equipos a analizar, se consideró el ranking de los 50 equipos de fútbol más valiosos de 2018 realizada por la revista Forbes, ya que se considera que toma indicadores importantes para su elección, y de cierta manera la afición puede cambiar más fácilmente o disminuir en un instante, aunque cuatro de los seleccionados se encuentran en los 10 primeros lugares de los 50 equipos con más aficionados.

La identidad de los identificadores de cada uno de lo equipos habla por sí solo y son ejemplo de algunos de los 14 parámetros que especialistas en la materia como Chaves y Bellluccia han declarado, de tal manera que se presentan a manera de análisis considerando la historia, contexto y esencia de equipos de fútbol como el Guadalajara -Chivas-, Monterrey, América, Santos Laguna y Tijuana.

\section{El fútbol en México}

El deporte es considerado como parte de la identidad de un país, de tal manera que si se piensa en Hockey inmediatamente se relaciona con Canadá, aunque también practiquen otros deportes, y en el caso de México, así como de otros países, el fútbol se puede considerar el deporte nacional, por lo tanto, es un reflejo de su cultura, pasión e identidad visual que se refleja en el comportamiento de los seguidores de los equipos y en la misma identidad de los equipos. De acuerdo a ESPN (2017) el fútbol es el deporte más popular en México, seguido por el box y en tercer lugar el béisbol.

Bajo el análisis de la agencia líder en el marketing deportivo Euroamerica Sport Marketing menciona que el negocio del balompié mexicano tiene un valor de cerca de los 67 mil millones de euros, posicionando a nuestro país dentro de las 10 economías del fútbol en el mundo (Huerta, 2018). Es por esto que tiene una gran importancia en nuestro país, además de que no requiere de mucho equipo ni vestimenta especial, y eso es lo que ha ayudado a la popularización de este deporte.

El origen del fútbol en México data en el año de 1898, cuando se funda el Orizaba Athletic $C l u b$, que en sus inicios era un club social y deportivo enfocado al Cricket, posteriormente comienza a realizar actividades de fútbol, años después en 1901 el Pachuca Athletic Club se funda con la intención de tener un club de fútbol. La Federación Deportiva de Occidente de Aficionados en Jalisco se crea en 1908, esta liga tiene gran éxito tal que se convirtió 
después en la primera liga mexicana, en ella participaron equipos como el Guadalajara y el Atlas (Revista Mira, 2018).

Es interesante conocer cómo se va transformando un deporte a lo largo del tiempo, pues esto ayuda a comprender cómo va adquiriendo importancia, fuerza e identidad nacional, al grado tal que para algunos aficionados se transforma en un ritual de fin de semana o en cada ocasión que su equipo entra a la cancha de juego.

Para convertirse en un deporte de competencia a nivel internacional, debe existir un organismo regulador, misión llevada a cabo por la Federación Mexicana de Fútbol -FEMEFUT-, fundada en 1927, de tal manera que en 1930 la selección de fútbol de México compite en su primer torneo internacional en la Copa Mundial de Fútbol de Uruguay. En 1961 se unió a la Confederación de Fútbol de Norte, Centroamérica y el Caribe -CONCACAF-, la cual tiene participación en América del norte, centro y el caribe, con torneos como la Copa Oro y la Liga de Campeones de la ConCACAF, además que los 41 países miembros disputan un torneo clasificatorio para participar por lugares en el Mundial de cada año. La FEMEFUT está afiliada a la Federación Internacional de Fútbol Asociación -FIFA- (Revista Mira, 2018).

La transformación del fútbol sigue teniendo presencia en México, tanto en cuanto a estructura como a la inclusión de las mujeres, de tal manera que, parafraseando a la revista Mira (2018), la Federación Mexicana de Fútbol realiza una reingeniería en todas sus categorías por lo que para 1994 crea la Primera División A, la cual toma el segundo nivel profesional en México, desplazando al tercer puesto a la Segunda División y al cuarto a la Tercera División. Para el torneo Apertura 2009 la Primera A fue reemplazada por la Liga de Ascenso, se redujo los equipos a 17 y un formato similar al usado en la primera división, esto con la intención de mejorar los niveles de crecimiento económico del balompié mexicano al tener dos torneos anuales y no uno solo como lo que ocurre en los torneos largos en Europa o Sudamérica. Cabe mencionar que la participación de las mujeres en este deporte, desde árbitros, asistentes, entrenadoras, traen consigo que para el año 2007 la federación, decide crear la Súper Liga Femenil, la cual tenía la participación de 24 equipos. Sin duda la estadística en los deportes es lo más representativo para evaluar su crecimiento en lo deportivo, así como en lo económico, los números del balompié mexicano son los siguientes:

- 57 son los clubes que han pasado por la Primera División.

- 4 son los equipos de se mantienen vigentes: Atlas, Veracruz, América y Chivas, siendo estos dos los que han disputado todos los partidos.

- 1, fue el torneo en el que participó Colibríes, en su efímero paso por el fútbol mexicano (Zamora, 2018).

En cuanto a la máxima expresión del balompié, el gol, grandes romperedes han participado en los torneos, los números en goles según Zamora (2018):

- 182 anotaciones, es el máximo número de goles en una temporada (1945-46). - 312 goles, es la marca de Evanivaldo Castro Cabinho, máximo anotador en Liga. 
- Un gol de Edwin Cubero le dio al Atlas el único campeonato de Liga en 1951. - 122 goles en 30 juegos fue el récord que estableció Atlante en la temporada 1945-46.

- 100 goles anotó Isidro Lángara, el primero en lograrlo.

- 9 segundos fue el tiempo que tardó Ricardo Chávez Medrano en anotarle a Chivas, en la temporada 1989-90. Que significó el gol más rápido en la historia de la Liga MX.

- Kantar Worldpanel agencia líder en el conocimiento del consumidor, estudió y organizó a la afición del fútbol en México en diferentes segmentos con lo que analizó sus hábitos de consumo (América retail, 2018), diferenciando 5 productos clasificados por el amor a la camiseta.
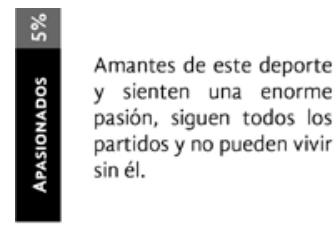

Siguen la mayor parte de los partidos, les encanta y es importante en sus vidas.

Les gusta pero su vida no gira en torno a él. De vez en cuando ven algún partido importante, por ejemplo el Mundial

El fútbol no les gusta ni les interesa en lo más mínimo, de hecho, no lo soportan. No ven los partidos ni siguen eventos importantes.

En estos hogares se encuentran integrantes con diferente grado de afición: apasionados, aficionados, apasionados $y$ haters.
I

| Formada por familias grandes | principalmente del norte del país,

| con niveles socioeconómicos bajos | y con niños menores de 12 años.

;

I En estos hogares hay presencia de hijos adolescentes, destacan en la I adquisición de productos de calidad.

\section{I}

Los interesados son familias medianas, es decir entre 3 y 4 integran| tes, son de nivel alto que viven en el noroeste y occidente del país. I

\section{;}

| Son familias pequeñas de 2 integrantes con amas de casa jóveI nes y sin hijos o son familias monoparentales.

\section{I}

I Son hogares de niveles socioeconómicos humildes con hijos mayores de edad.
Este grupo es quien va más de compra, 256 veces al año.
Son quienes menos adquieren productos de consumo inmediato, pero si gastan en tiendas de convivencia.

Compran principalmente en el canal moderno, en especial en hiper y supermercados.

\section{। ,}

Los hogares que registran un menor gasto en productos de consumo masivo son ellos.

I

Los hogares mixtos son quienes más gastan en marcas económicas.

Tabla 1. Clasificación de aficionados al fútbol. Fuente: Creación propia basado en América retail (2018, s/p). 
Debido a que los aficionados se han estado cultivando por este apasionante juego de pelota, la revista FORBES en su análisis denominado Los 50 equipos de fútbol más valiosos de América en 2018, declara que "tres cuartas partes de los 50 clubes mejor valuados en el Continente Americano son instituciones brasileñas, estadounidenses y mexicanas. En conjunto, suman un valor de 7,324 millones de dólares (mdd)"(Pérez, 2018, s/p).

$\mathrm{Al}$ analizar el ranking de esta clasificación, es evidente que en los lugares 6 y 7 se encuentran los equipos monterrey y chivas, además 11 clubes de 18 que forman la Liga MX están entre los 50 equipos más valiosos del continente de acuerdo a este estudio, lo que representa al fútbol mexicano como una gran economía de fútbol a nivel mundial. Algunos aspectos que consideran para la definición de estos lugares es el valor de los jugadores que pertenecen a la institución, ya que no toma en cuenta aquellos que se encuentran en préstamo; el valor de la marca y costo del estadio si es que el club es dueño del inmueble.

\begin{tabular}{|c|c|c|c|}
\hline Puesto & EQUIPO & VALORADO & \\
\hline 6 & Chivas & $297.1 \mathrm{mdd}$ & \\
\hline 7 & Monterrey & $281.8 \mathrm{mdd}$ & \\
\hline 15 & América & $174.8 \mathrm{mdd}$ & \\
\hline 16 & Santos & $164.6 \mathrm{mdd}$ & \\
\hline 19 & Tijuana & $140 \mathrm{mdd}$ & \\
\hline 23 & Pumas & $125.3 \mathrm{mdd}$ & \\
\hline 24 & Toluca & 116 mdd & $\begin{array}{l}\text { Tabla 2. El puesto en } \\
\text { que se encuentran los }\end{array}$ \\
\hline 28 & Tigres & $93 \mathrm{mdd}$ & $\begin{array}{l}\text { diez primeros equipos } \\
\text { de fútbol mexicano en el }\end{array}$ \\
\hline 35 & Cruz Azul & $78.1 \mathrm{mdd}$ & $\begin{array}{l}\text { ranking de los } 50 \text { equipos } \\
\text { de fútbol más valiosos de }\end{array}$ \\
\hline 39 & Pachuca & $68 \mathrm{mdd}$ & $\begin{array}{l}\text { América en 2018. Fuente: } \\
\text { Pérez }(2018, \mathrm{~s} / \mathrm{p} .) \text {. }\end{array}$ \\
\hline
\end{tabular}

A pesar de que los cinco primeros equipos mexicanos que aparecen en el ranking no son los que más aficionados tienen, si se encuentran algunas coincidencias con respecto a su popularidad, pues de acuerdo a Rendón (2019) los equipos que más han ingresado aficionados a sus estadios son el América con 9.7 millones, seguido por el Monterrey con 8.3 millones, las Chivas quedan en cuarto lugar con 6.5 millones, en séptimo Santos Laguna con 5.2 millones, mientras que Tijuana no aparece en este listado. 


\begin{tabular}{|c|c|c|c|}
\hline PUEST & EQUIPO & VALORADO & \\
\hline 1 & América & $9.7 \mathrm{mde}$ & \\
\hline 2 & Monterrey & $8.3 \mathrm{mde}$ & \\
\hline 3 & Tigres & $8.2 \mathrm{mde}$ & \\
\hline 4 & Chivas & 6.5 mde & \\
\hline 5 & Pumas & $6.2 \mathrm{mde}$ & \\
\hline 6 & Atlas & $5.4 \mathrm{mde}$ & \\
\hline 7 & Santos & $5.2 \mathrm{mde}$ & \\
\hline 8 & Morelia & 5 mde & $\begin{array}{l}\text { Tabla 3. Los } 10 \text { equipos } \\
\text { mexicanos con mayor }\end{array}$ \\
\hline 9 & Cruz Azul & 4.707 mde & $\begin{array}{l}\text { afición, de acuerdo a los } \\
\text { millones de espectadores } \\
\text { (mde). Fuente: Creación }\end{array}$ \\
\hline 10 & Pachuca & 4.771 mde & $\begin{array}{l}\text { propia basado en Rendón } \\
(2019, \mathrm{~s} / \mathrm{p} \text {.). }\end{array}$ \\
\hline
\end{tabular}

Sin duda el fútbol en México es parte de la cultura del mexicano, una tarde de deporte para disfrutar un partido en la televisión, o verlo jugar en los campos del barrio, o incluso ver a los niños apasionados que imitan a sus héroes deportivos, son parte de la vida del mexicano.

\section{La identidad deportiva a través del diseño}

Para comprender de qué manera el diseño gráfico aporta a la identidad deportiva de un país, es necesario tener una postura acerca de la identidad, sus características y la manera en que interactúa con el deporte, en este caso con el fútbol, es por eso que se toma en cuenta la definición de identidad de Cortina (2007) quien dice que es

Lo que el ente, el ser, el objeto, el cuerpo químico, el vegetal, el animal o la persona física, empresa o institución, ES EN SÍ MISMO. Lo que le hace ser lo que es, igual solamente a sí mismo, irrepetible y absolutamente distinto de otro ser (p. 63). 
De esto se desprende que se definen diferencias que identifican y representan a cada ente, que se complementa con la reflexión que hace Del Val (2004).

Cuando reflexionamos en torno a la identidad, tendemos a construir un edificio analítico en el cual desaparece la complejidad identitaria y sus niveles (individual, familiar, de banda, de colonia, de ciudad, de región, de país, de clase, de ocupación, de adscripción religiosa, política, nacional, etcétera) (p. 13).

De tal manera que ese ente puede ser el cúmulo de características de diferentes aspectos que conforman a un ente mayor, es decir, un listado de características conforman la identidad de un equipo de fútbol, que a su vez, ese listado de características de todos los equipos conforman al fútbol mexicano y aportan a la identidad de un país.

Existen elementos que hacen que una empresa o institución tenga una identidad que lo hace diferente a cualquier otra, y que algunos de estos factores son "su origen, quién o quienes la fundaron, cuál es su filosofía bien diferenciada y real, sus valores, su historia, su situación financiera a lo largo del tiempo, sus planes actuales, etcétera" (Cortina, 2007, p. 67). Es por esta razón que es necesario conocer un poco más sobre los antecedentes y contexto en que se han desarrollado, en este caso, los equipos de fútbol.

Toda identidad, de acuerdo a Chaves y Belluccia (2008) se presenta básicamente en dos variantes: "el tipo de signos y sus variantes: cromáticas, dimensionales, sintácticas, etcétera; el tipo de piezas y soportes: aplicaciones heterogéneas que permitan verificar los rendimientos reales de los signos 'en acción' (legibilidad, reproductibilidad, etcétera)” (p. 108), con esto se puede definir que la proyección de la identidad se genera desde los identificadores de los equipos hasta las piezas que se derivan de él o que son necesarias como el uniforme, bandera, mochilas, calcomanías y todo tipo de artículos que ayuden a generar un sentido de pertenencia de los aficionados.

Existen catorce parámetros que son de naturaleza comunicacional, que ayudan a que la identidad y sus piezas gráficas sean congruentes y que cumplan con un objetivo tanto en la generación de los signos y sus variantes como con las piezas y soportes que complementarán la identidad gráfica a realizar, y que servirán como base para el análisis de los identificadores de los 5 equipos mejores rankeados en el listado de los 50 equipos de fútbol más valiosos de América Latina que ya se han mencionado (Chivas, Monterrey, América, Santos, Tijuana). El primero es la Calidad gráfica genérica, que "es la que logra seleccionar el o los lenguajes adecuados e interpretarlos con absoluto dominio de sus principios" (Chaves y Belluccia, 2008, p. 42), es decir, elegir una paleta tipográfica, paleta de color, abstracciones que sean de calidad, congruentes y armónicas que representen una unidad con respecto al estilo.

El segundo es el ajuste tipológico que hace referencia a que se pueden generar, además del logotipo, otras variedades de identificadores como "isotipos, monogramas, mascotas, colores y gráficas complementarias, [...] cada necesidad identificatoria concreta definirá qué tipos sígnicos son absolutamente obligatorios" (Chaves y Belluccia, 2008, p. 44), de tal manera que debe existir una congruencia entre ellos, por ejemplo, en los equipos de fútbol es sumamente común que existan mascotas.

El tercero trata de la corrección estilística que implica que "interpretar un mensaje no es solo detectar su contenido semántico sino fundamentalmente su estilo” (Chaves y Belluc- 
cia, 2008, p. 45), y ese estilo se deberá reflejar en todo lo que tenga relación con el club futbolístico en cuestión.

El cuarto es la compatibilidad semántica. "La única condición semántica universal es la compatibilidad: el signo no debe hacer referencias explícitas a significados incompatibles o contradictorios con la identidad de la organización" (Chaves y Belluccia, 2008, p. 47), es decir que la escencia del equipo se debe reflejar gráficamente.

El quinto habla de la suficiencia: "La suficiencia indica que los signos son los necesarios [...] para satisfacer todos los usos identificatorios del caso" (Chaves y Belluccia, 2008, p. 47), es decir, ni más ni menos, sino lo suficiente para comunicar lo acordado.

En el sexto, la versatilidad se hace presente cuando "los signos deben ser diseñados atendiendo a un rendimiento parejo en todos los niveles de discurso, sin pérdida de su uniformidad $[\ldots]$ si necesito acercarme a un nuevo segmento, debo reformular mi lenguaje publicitario, mi oferta, mi producto, mi servicio, pero no distorsionar mi logotipo" (Chaves y Belluccia, 2008, p. 49), eso implica que un identificador puede ajustarse a diversos mercados y piezas gráficas.

El séptimo es la vigencia y menciona que los identificadores "acompañan toda la trayectoria de una organización, deben ser de una vigencia no inferior a la vida de la organización” (Chaves y Belluccia, 2008, p. 51), es decir que no serán fruto de una moda, sino que en verdad refleje al escencia del equipo para que pueda prolongarse su vida lo más posible y generar una identificación a través de los años.

El octavo es la reproducibilidad que hace referencia a que "a mayor heterogeneidad de medios corresponderá un desafío al rendimiento 'físico' de los identificadores" (Chaves y Belluccia, 2008, p. 52), ya que deben adaptarse a piezas de diseño muy diferentes, con características muy particulares.

El noveno corresponde a la legibilidad e "indica el grado de reconocibilidad visual de los rasgos esenciales del signo" (Chaves y Belluccia, 2008, p. 52), que se puedan identificar con facilidad, que no cree confusión y que al colocarse en diferentes tamaños no tenga problemas para su lectura.

El décimo es la Inteligibilidad, que "es la capacidad que tiene un signo para ser comprendido en las condiciones normales de lectura [...] es la claridad o certidumbre con que el público decodifica el sentido del signo observado" (Chaves y Belluccia, 2008, p. 53), es decir que, si es una flor, denote que es una flor sin generar confusión.

El undécimo habla de la pregnancia, mejor conocida como la "capacidad que tiene una forma de ser recordada" (Chaves y Belluccia, 2008, p. 54), y que cuando se hable de un equipo de fútbol, inmediatamente se venga a la mente el identificador correspondiente, sin confusión. El duodécimo es la vocatividad, conocida como la "capacidad del signo para atraer la mirada [...] [sus recursos son] agresividad del color, dinamismo de la forma, expresividad de los iconos, protagonismo por tamaño o proporción, etcétera" (Chaves y Belluccia, 2008, p. 55), es decir, que sea llamativo, claro que sin dejar de lado la correspondencia con la escencia de, en este caso, el equipo de fútbol por representar.

El décimo tercero corresponde a la singularidad. Se sabe que "se es singular sólo en relación con otros [...]. Se debe ser tan singular como lo exija la propia identidad" (Chaves y Belluccia, 2008, p. 56), es decir, que no se parezca a otros, pero que si se identifique como parte de su categoría, aunado a la escencia del equipo que debe reflejar. 
El décimo cuarto y último habla de la declinabilidad, que "es un parámetro que se aplica solo para ponderar el cumplimiento del grado de codificabilidad indispensable" (Chaves y Belluccia, 2008, p. 58), es decir que no parezca lo que no es.

Con base en estos catorce parámetros se realizará el análisis de los identificadores de lo equipos Chivas, Monterrey, América, Santos y Tijuana, de los cuáles se presentará un poco de su historia y contexto, para poder entender mejor su esencia y su representatividad gráfica.

\section{Chivas: $100 \%$ mexicano}

En primer lugar, tenemos al club deportivo Guadalajara mejor conocido como Chivas, este equipo fue fundado el 8 de mayo de 1906 y es uno de los equipos con más campeonatos en la historia del futbol mexicano, en el 2003 las Chivas comienzan un proceso de renovación cuando fue comprado por el empresario Jorge Vergara, lo cual despuntó al equipo en la afición mexicana, convirtiéndolo en el equipo más popular de México y a las Chivas rayadas del Guadalajara en una marca exitosa en el mercado.

En 1908 se conforma de manera oficial como Club Guadalajara, y era necesario contar un elemento que los identificara y los hiciera destacar, en un principio solamente utilizaban las iniciales C. G. -Club Guadalajara-, la C era representada por una silueta rojo oscuro que da la impresión de ser la representación de una serpiente la cual rodea a la G de color blanco cuyo peso y tamaño son en proporción mayores, contaba con un envolvente circular de color rojo, por el peso histórico de este identificador, fue seleccionado como el que se utilizaría para el uniforme conmemorativo del centenario de la institución.

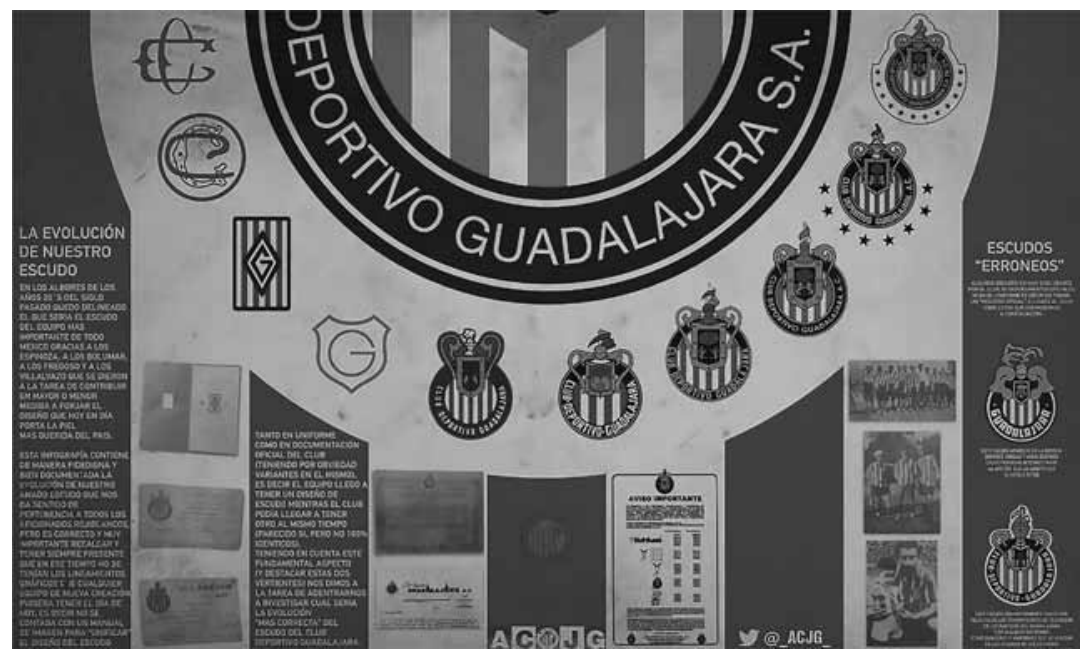

Figura 1. Evolución gráfica del escudo del club deportivo Guadalajara (Asociación de coleccionistas de Jerseys del Guadalajara, 2018). 
La evolución gráfica de los elementos identificadores del club va desde la utilización de las iniciales rodeadas con un envolvente que va desde lo circular, rectangular hasta comenzar a tomar la forma de escudo, siendo que hasta la tercera modificación del signo se incluyen los colores rojo blanco y azul, así como las emblemáticas líneas rojiblancas que hasta el día de hoy son elemento altamente posicionado en la mente tanto de los aficionados de este equipo como en el público en general, es claro como el símbolo va madurando a partir de que se genera el primer escudo con las características gráficas y conceptuales que conserva hasta la fecha.

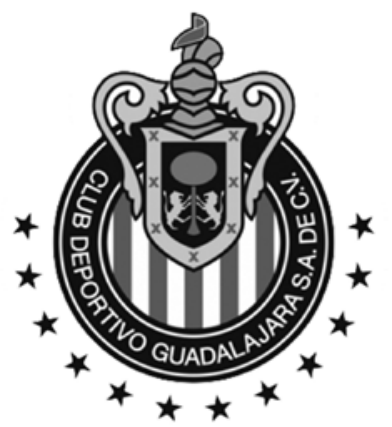

Figura 2. Escudo oficial del club deportivo Guadalajara en la actualidad (Mejoress, 2019).

El escudo actual del Club Guadalajara se crea en la campaña 1923-1924 y se atribuye a jugadores y directivos del mismo club, como Villalvazo, Everardo Espinoza y su hermano José El Tata, además de Ángel Bolunar, se aprecia cómo es que el escudo contiene las líneas rojiblancas que desde inicios del equipo forman parte del uniforme y son inspiradas en la camiseta del equipo de Brujas de Bélgica, cuadro donde jugó el fundador del Club Evereart, se integra también el escudo de armas de la ciudad de Guadalajara, las aspas simbolizan el estandarte o guión del caudillo invicto en los combates, la lanza la fortaleza con prudencia, la orla se concebía como insignia de gran valentía. Simboliza el león a un espíritu generosamente guerrero, adornado de las cualidades de vigilancia, dominio, soberanía, majestad y bravura. El pino es el símbolo de perseverancia (Ideas frescas, 2019). El escudo se complementa con la leyenda Club Deportivo Guadalajara, y en la actualidad se le agregó S.A. de C.V. en un círculo de color azul que rodea un estampado con 5 barras verticales en color rojo y 6 en color blanco que representan los colores de la institución utilizados en el uniforme. Al final 11 estrellas rodean al círculo representando los $11 \mathrm{cam}$ peonatos que el Guadalajara ha logrado en el máximo circuito del fútbol mexicano la Primera División.

Se puede considerar el éxito de Chivas como marca ya que el equipo es un fenómeno social en México, representa una tradición y podría decirse sin miedo a equivocarnos que 
es una segunda selección mexicana, esto se representa en su política de integrar solo mexicanos en el equipo, un elemento que le ha dado un signo distintivo al equipo desde su creación. Chivas es un icono para los aficionados que lo siguen, representa un estilo de vida, propone nuevas estrategias para satisfacer las necesidades y deseos de fútbol de los aficionados y consumidores de este equipo.

El club deportivo Guadalajara, logró un salto comercial al convertirse en una empresa redituable para el empresario Jorge Vergara, con esto se posibilitó la oportunidad de negocios para aficionados y empresarios ya que comenzó a otorgar franquicias para la venta de ropa y artículos explotando la imagen y posicionamiento de la marca en la sociedad mexicana.

Un equipo de fútbol es un producto que tiene más en común con el ocio, el espectador deportivo busca espectáculo, diversión, entretenimiento, pasión, Chivas logró empatar la visión comercial con el sentimiento, lo cual le facilita la inserción de sus productos y servicios en el mercado nacional e internacional, el modelo de negocio del equipo ha pasado de una industria principalmente local a un negocio global que se inserta en la industria del entretenimiento.

Se considera que el nombre de Chivas logra un $98 \%$ de reconocimiento entre los mexicanos y latinoamericanos, la identidad que logra el equipo al integrar únicamente a jugadores mexicanos en su plantilla es un elemento identificador que lo vuelve emblemático para los aficionados e incluso para los que no son aficionados de este equipo, pero gustan de este deporte.

\section{Monterrey: La Pandilla}

En segundo lugar en la lista de los equipos más populares en el 2018, tenemos al Club de futbol Monterrey, sus inicios son en 1945, era un momento difícil para el país ya que pasaba por una época de guerra y crisis, además el club no tenía los mejores pronósticos al ser el béisbol el deporte más importante para los regiomontanos en ese momento, es por esto que la idea de un equipo de fútbol no era lo más conveniente en el momento. A pesar de las preferencias sociales, políticas y contextuales de la sociedad, quienes iniciaron este proyecto siguieron adelante con la idea y aprovecharon que en ese momento el estado de Nuevo León obtuvo el reconocimiento de Primera Fuerza Nacional, la euforia colectiva fue lo que les hizo tomar la decisión final para fundar finalmente el Club de Fútbol Monterrey, S.A. de C.V. (Club de Futbol Monterrey Rayados, 2018).

El primer juego del nuevo equipo se llevó a cabo el 19 de agosto de 1945 en el parque de béisbolCuauhtémoc en donde lograron la victoria ante el San Sebastián de León con un marcador de uno por cero, el gol fue anotado por el jugador José Che Gómez, el equipo no dejó de buscar el ascenso en los años posteriores a su debut hasta que el 25 de Diciembre de 1955, jugó ante el mismo rival y al vencerlo, lo logró en la temporada 55-56, el primer juego del ahora equipo en primera división tiene lugar el 8 de julio en Zacatepec, Morelos (Club de Futbol Monterrey Rayados, 2018). 
El nombre de batalla del equipo fue sujeto a cambios, algunos de ellos fueron, Sultanes, Gitanos, Norteños, Nuevoleoneses y Regios hasta llegar a los apodos más populares con los que ahora los conocemos que son, La Pandilla y Rayados.

El sobrenombre más popular y que es el que identifica de forma significativa al equipo ante la afición es el de Rayados, fue apoyado por algunos periodistas como Ramón Oviedo, Cesar M. Saldaña y José Reyna (Club de Futbol Monterrey Rayados, 2018).
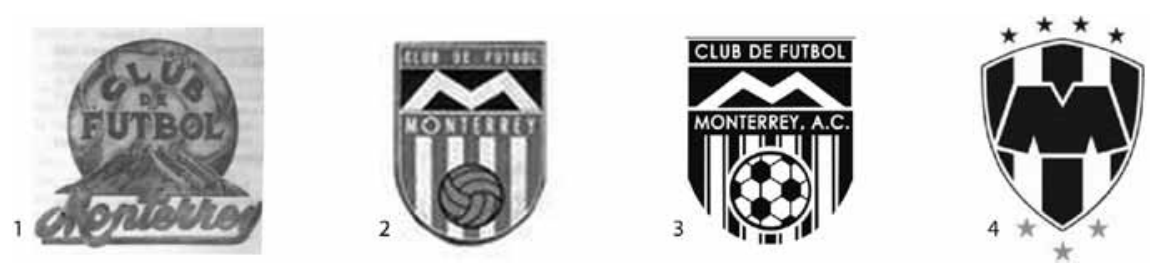

Figura 3. Evolución del escudo del equipo Monterrey (Milenio, 2019) ${ }^{1 y^{4}}$, (Fuentevilla, 2009)2,$($ Preciado, $2018)^{3}$.

Hablando de la evolución gráfica que ha sufrido el signo identificador desde sus inicios, se puede observar que en un principio utilizó el emblemático cerro de la silla, integrando un envolvente en la parte posterior que hace referencia una puesta de sol en el horizonte combinando diferentes estilos tipográficos dentro del mismo identificador, en los posteriores signos identificadores se puede apreciar cómo es que se conserva en la representación del cerro de la silla y a la vez simulando la letra $M$, unificando la tipografía en una sola fuente de caja alta. Como se puede observar es el primer momento en el cual aparecen las emblemáticas franjas azules y blancas, además de la integración de un balón de fútbol que en primer momento puede dar la impresión de ser de voleibol, en la siguiente edición se propone la forma más clara de un balón de fútbol, elemento que es omitido en el rediseño que continúa vigente hasta la fecha. 


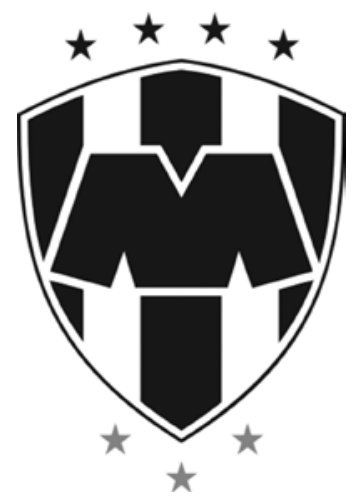

Figura 4. Escudo oficial del Club Deportivo Monterrey (Preciado, 2018).

En 1989 tras la venta del equipo, se logró un rediseño al escudo el cual sigue vigente hasta la fecha, el signo identificador actual del equipo Rayados de Monterrey su elemento principal es la letra $M$ es la representación abstracta del Cerro de la Silla, uno de los mayores iconos del estado de Nuevo León, específicamente de Monterrey, la cual por sí misma hace alusión a al nombre del equipo sin tener que hacer uso del logotipo, además de tener la forma la camiseta del equipo se conservan las franjas azul y blanco, las cuales se ven desde los identificadores anteriores, específicamente tres franjas azules y dos blancas. Es un identificador muy limpio y claro, moderno en cuanto a su concepto, y que alrededor del mismo se presentan las estrellas correspondientes al número de títulos obtenidos por el club. El club deportivo Monterrey cuenta con una de las mejores aficiones a nivel nacional, esto es reconocido por la prensa mexicana, sus jugadores y directivos, es una de las aficiones con mayor porcentaje de asistencia, así mismo se ha vuelto común la asistencia de los aficionados a los entrenamientos a puerta abierta en el Estadio Tecnológico, llegando a tener entradas de hasta 20 mil personas en las prácticas(Liga MX, 2019a).

\section{América: Azul - crema}

En tercer lugar se encuentra el legendario equipo América, que en el año de 1961, cuando Rafael Garza Gutiérrez y su primo Germán Nuñez Cortina, adolescentes y estudiantes del Colegio Mascarones quienes jugaban fútbol todos los días como actividad recreativa, decidieron crear un equipo con los mejores elementos de su escuela, a los cuales asistieron 13 jóvenes estudiantes a la primera cita (Club de Futbol América, 2019).

El líder del proyecto, Rafael Garza Gutiérrez, fue el más entusiasta, quien sorprendió a los asistentes llegando prácticamente uniformado, con una vieja camisa color crema, desgastada, de mangas largas y botones al frente, un pantalón corto azul marino y calcetas largas 
azul marino, es ahí donde nace el legendario uniforme que hasta el día de hoy es emblemático tanto para el club, como para la afición del equipo.

A la par de este suceso en otro centro escolar Marista existía un equipo de reciente formación, en el cual sus principales jugadores estaban desanimados ya que no alcanzaban a cubrir la plantilla mínima reglamentaria para un partido de fútbol, así que su entrenador, Eugenio Cenoz, propuso una junta con el conjunto encabezado por Rafael Garza Gutiérrez, para realizar la propuesta de fusionar ambos conjuntos como un solo equipo, y es así como se da el potencial nacimiento del Club América.

El día 12 de octubre de 1916, en los llanos de la Condesa se reunieron ambas escuadras para discutir el nombre del equipo que los unificaría, puesto que para el uniforme ya habían decidido adaptar el azulcrema que presentó Rafael Garza Gutiérrez, por considerarlo hermoso y diferente a los ya existentes.

Fue en la misma fecha cuando se decidió cuál sería el nombre de la recién formada escuadra, fueron muchas las propuestas, pero al final Pedro el Cheto Quintanilla sugirió el nombre de América ya que esa fecha coincidía con el aniversario del descubrimiento del continente. Fue el mismo Quintanilla, el cual tenía destacadas habilidades para el dibujo, quién presentó la propuesta con un bosquejo del continente americano incluyendo desde ese momento los colores azul-crema además de incluir las iniciales del equipo recién conformado: la $C$ de club y la $A$ de América.

Fue así como el 12 de octubre quedó establecido oficialmente el nacimiento del club y también los históricos colores con los que hace poco más de cien años se ha identificado el equipo, a partir de 1920 inicia la época de esplendor deportivo del club, la cual hasta la fecha sigue conservando los elementos distintivos hasta la actualidad (Club de Futbol América, 2019).

El signo identificador del club deportivo tiene como características principales el uso de una representación figurativa no realista del continente americano, el cual está rodeado por la representación de un balón incluyendo las siglas $C$ y $A$, características que están presentes desde la década de los 50 , aunque el escudo ha pasado por varias modificaciones en su estructura gráfica por poco más de cien años.

En la primera versión de escudo se puede observar cómo es que la gama cromática era menos saturada, además de que el envolvente hace total referencia al planeta. Para 1918 el club sufre un cambio de nombre y por tanto también el signo identificador, en el cual se integran las iniciales del nuevo nombre: Club Unión, aunque la gama cromática es respetada y la estructura también conserva las mismas características. 


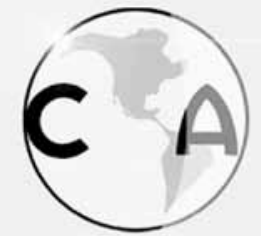

จำด

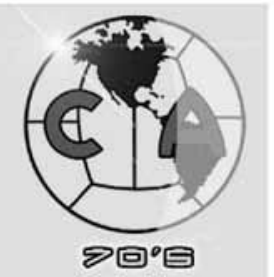

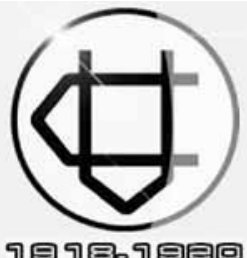

จตจ๒จตย回

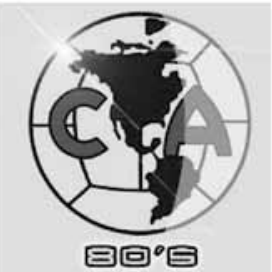

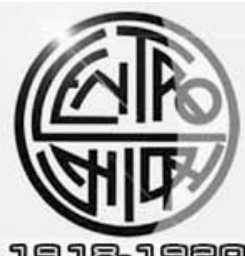

จตจยจตอต

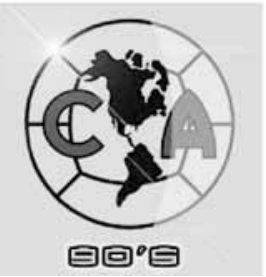

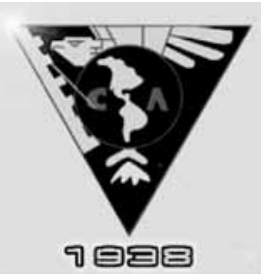

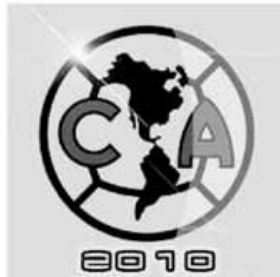

Figura 5. Evolución histórica del escudo del Club de Fútbol América (Galván, 2019, s/p.).

Dentro del mismo periodo 1918 a 1920 se puede observar cómo es que el escudo del Club Unión es modificado y se integra una composición tipográfica en donde la tipografía adquiere un valor icónico al ser utilizada como imagen, lo cual da mayor importancia a este elemento, sin embargo, la legibilidad del mismo es complicada, lo cual causa que no sea pregnante ni de impacto para la afición sufriendo nuevamente modificaciones significativas en 1938.

La necesidad de refrescar la imagen del club deportivo lleva a los directivos del equipo a cambiar el escudo, aunque esta modificación duraría un corto periodo de tiempo ya que el trazado original fue encerrado en un triángulo, respondiendo a las tendencias de la década de los 40's, situación que para esas fechas fue común en los equipos deportivos e incluso se elaboraban banderines con esta forma para el intercambio entre rivales deportivos. En este signo identificador se puede observar que aparece un águila, esto es curioso ya que, para ese tiempo, el club todavía no obtenía el mote de las Águilas del América, lo cual es considerado una casualidad o una premonición. Este diseño solamente fue utilizado una temporada y después se regresó al diseño base.

En 1959 el equipo fue adquirido por el empresario Emilio Azcárraga, quién decidió inyectar el capital necesario para que el equipo de primera división se convirtiera en uno de los más emblemáticos del futbol mexicano.

En cuanto a la evolución del escudo se regresó a los elementos básicos en 1970, integrando elementos de comunicación más sólidos que le han dado la pregnancia necesaria para posicionarse en el consciente colectivo, tanto de sus aficionados como del público en general. Se transformó el círculo que rodea al continente americano en un balón, lo cual le dio un sentido más claro de que se trataba de un equipo de fútbol soccer, así mismo se realizaron 
ajustes a la representación del continente logrando una mayor calidad gráfica, el color amarillo y el azul se presentan con mayor saturación, proporcionando un mayor impacto visual en la composición gráfica. Se integró un tercer color, el rojo en los elementos tipográficos, los cuales también sufrieron modificaciones en el peso y el trazo. Esta versión sentó las bases para lo que hoy en día conserva el identificador.

El cambio que sufre el signo en la década de los 90's, es más de forma y recursos gráficos concretos, una mayor proporción del continente, con un acercamiento más evidente a México, también se centra de manera que queda en medio de los elementos tipográficos dando un mayor equilibrio y visual.

En los años noventa se reafirma el concepto que se manejó anteriormente, pero se estiliza el signo identificador para facilitar la reproductibilidad y darle más pregnancia. Se omite la línea central del círculo para evitar efectos visuales nocivos o que puedan crear confusión, también se modificaron los elementos tipográficos haciendo los ajustes ópticos necesarios para mejorar los rasgos de las letras y facilitar tanto su reproductibilidad como su rendimiento.

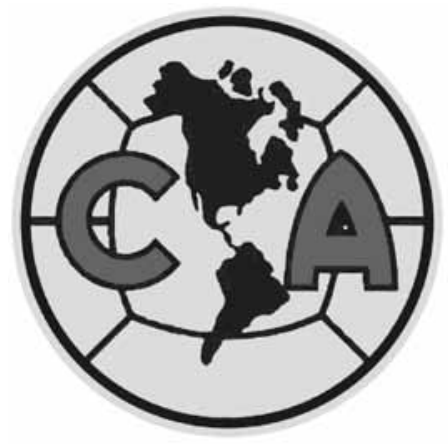

Figura 6. Escudo oficial del Club deportivo América en la actualidad (Club de Futbol América, 2019).

La última modificación realizada dio lugar en el 2010, con un identificador más maduro, un cuidado mayor en los detalles finos, el trazo es mucho más grueso lo cual facilita la adecuada jerarquización de los elementos visuales, así como la reproductibilidad en los diferentes sustratos y sistemas de impresión. también se genera un mejor contraste, logrando que los elementos que integran el escudo estén mejor sintetizados logrando así una mayor calidad gráfica.

Es notable como el club deportivo América ha logrado evolucionar su imagen desde sus inicios, pero sin perder la esencia y la historia que viene dando soporte para generar una mayor identificación y sentido de pertenencia con la afición. 


\section{Santos Laguna: poco tiempo, mucha historia}

El equipo que se encuentra en cuarto lugar dentro de la lista de Forbes es un caso singular ya que, a pesar del poco tiempo de haber sido conformado, ha logrado tener mucha historia. Este club con apenas 35 años de historia a logrado convertirse en un elemento clave de la identidad de la Comarca Lagunera, además de uno de los modelos de negocio y deportivo más importantes y exitosos de México (Club Santos Laguna, 2019a).

En la década de los ochenta, el Instituto Mexicano del Seguro Social (IMSS) se encontraba en uno de sus mejores momentos y fue por esto que le dio un gran impulso al deporte en México, propició la adquisición de varias franquicias del fútbol mexicano, sin sospechar que una de ellas se convertiría en uno de los equipos con mayor éxito y aficionados en el balompié nacional (Club Santos Laguna, 2019a).

El origen del Club Santos Laguna fue en 1982 cuando la franquicia de tuberos de Veracruz es adquirida por el IMSS, el equipo se ubicaba en Santa Cruz, Tlaxcala, lugar que le daría el nombre representativo al equipo lagunero, ya que a los habitantes de esa localidad se les conoce como Santos. Las negociaciones en la temporada 1982-1983 el seguro social cerró negociaciones en Durango e instaló una franquicia en la laguna para esa misma temporada, el cual se coronó en los torneos nacionales ganando el premio de contar con una franquicia en la segunda división $B$, el primer partido de primera división se jugó en el Estadio Corona contra Bachilleres.

El ascenso a segunda división A se dio 12 meses después, hasta que en la temporada 88-89 se concreta la compra de los Ángeles de Puebla y es cuando el equipo Santos tiene presencia en la primera división (Club Santos Laguna, 2019a).

El escudo del equipo de la Laguna ha sufrido un gran número de cambios desde sus inicios hasta la actualidad, a pesar de esto ha logrado tener pregnancia dentro de la afición mexicana.

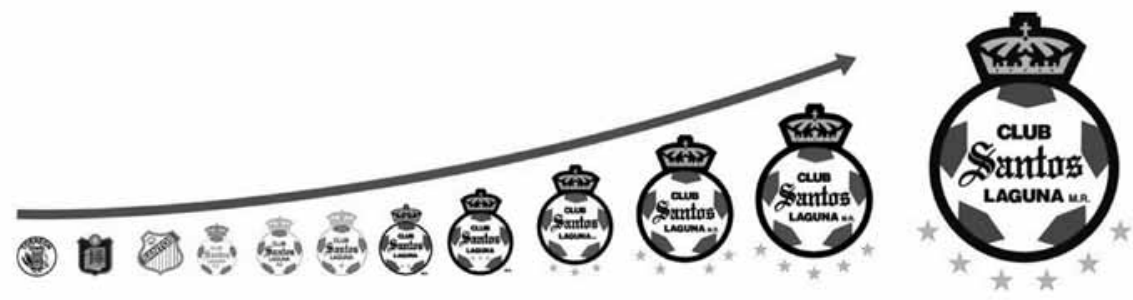

Figura 7. Evolución histórica del escudo del club Santos Laguna (Club Santos Laguna, 2019b). 
El primer escudo del equipo tuvo una similitud con el de su homónimo brasileño, pero en 1983 fue modificado con el verde que hasta la fecha es el color institucional, en esa versión del signo identificador se incluyen también las siglas del Instituto Mexicano del Seguro Social (IMSS) el cual en esas fechas era el propietario del equipo.

La nueva etapa del equipo, el grupo cervecero Modelo vio oportunidad de crecimiento tanto social como financiero y decidieron adquirir al equipo, invirtieron en jugadores de jerarquía y así fue como comenzó una nueva etapa del equipo que logró calificar por primera vez a la liguilla para lograr llegar a la final y quedar en segundo lugar.

Es durante este periodo del 1986 a 1995 cuando renovaron su imagen, creando un nuevo escudo y dando el primer esbozo de su evolución hasta el actual. Consistía en la representación de un balón con cinco pentágonos, el nombre del club al centro y la corona sobre él, misma que fue incluida tras la adquisición del grupo modelo. Después de su primer campeonato, una estrella se colocó dentro del balón verde. Los colores se modificaron en el 2001 para lograr mayor contraste e incluir un contorno negro con mayor puntaje. La corona se modificó, ya que la que había sido utilizada hasta ese momento correspondía a la de una cerveza del grupo modelo, fue por esto que se decidió dar su propia personalidad a la corona que representa al equipo, sus raíces y personalidad.

En el año 2008 y con una nueva directiva al mando, se realizó una nueva modificación al escudo, colocando las estrellas que hacen referencia a los campeonatos fuera de la representación del balón. Se realizaron modificaciones que aumentan la calidad gráfica del signo identificador, pero siempre respetando la esencia del mismo, de tal manera que el contorno negro ya no solamente rodea la figura que representa al balón, sino que se desarrolló una silueta que contornea a todo el escudo sin incluir las estrellas, lo que le da más fuerza. Desde ese momento no ha vuelto a sufrir cambios en su estructura, solo se han agregado las estrellas, que hoy en día ostenta seis, que representan los campeonatos que ha logrado el club a lo largo de su estadía en la primera división del futbol mexicano.

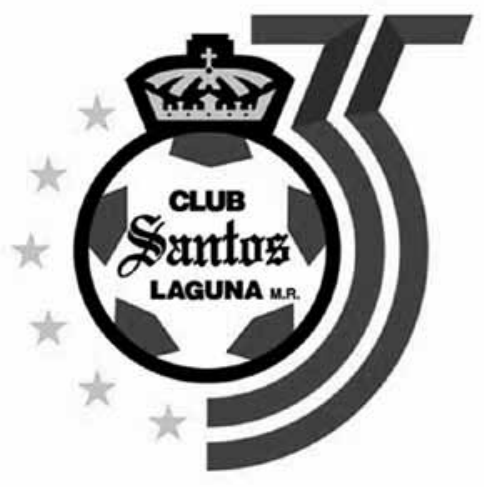

Figura 8. Escudo conmemorativo del 35 aniversario del club Santos Laguna (Liga MX, 2018). 
Dentro de las estrategias de comunicación del club deportivo, se ha generado un signo identificador especial el cual fue diseñado para conmemorar el 35 aniversario del Club, hecho que potencializa la identificación de la trascendencia del club ante los aficionados laguneros.

\section{Club Tijuana: el hermoso}

En la sexta posición se encuentra el Club Tijuana, también conocido como Xolos, este es el club más joven de todos los que están presentes en la lista de Forbes, referentes al futbol mexicano, apenas fundado en el 2007 en tan solo cuatro años logró ascender a la primera división, desde sus inicios los directivos de la escuadra, Jorge Alberto Hank Inzunza y Gog Murguía, presidente y vicepresidente respectivamente cimentaron bases sólidas para que el equipo fuera de primera división, se crearon las fuerzas básicas y la construcción del Estadio Caliente, hasta el día de hoy el territorio de los Xolos mantiene un constante crecimiento tanto en la primera división como en las divisiones juveniles y en la tercera división (Club Tijuana Xoloitzcuintles de Caliente, 2019).

El proyecto Tijuana, como fue llamado por los directivos, apuesta por el Centro de Iniciación Xoloitzcuintles que es una escuela de fútbol infantil que ha crecido de manera significativa, al punto de abrir nuevos planteles en varios lugares de la frontera, así como en territorio Californiano. Otro de los pilares del proyecto es la Escuela Nacional de Directores Técnicos es el Campus Xoloitzcuintles que ha capacitado una buena cantidad de nuevo entrenadores que obtienen la oportunidad de insertarse en el fútbol mexicano (Club Tijuana Xoloitzcuintles de Caliente, 2019).

En tan solo doce años desde su fundación el Club Tijuana ha logrado generar un arraigo importante para la ciudad del mismo nombre. Pasaron tan solo cuatro años desde la creación del equipo cuando se llegó a primera división y fue en su quinto aniversario cuando los Xolos le dieron a Tijuana su primer campeonato en la primer división del balompié mexicano.

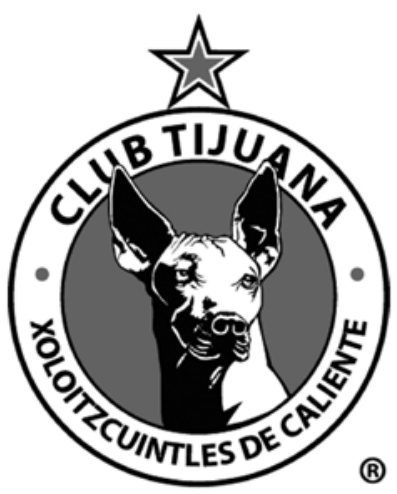

Figura 9. Escudo del Club Tijuana (Liga MX, 2019b). 
El escudo de la escuadra de Xolos tiene una historia peculiar, años antes. El padre del directivo Jorge Hank recibió un perro Xoloitzcuintle como regalo de manos del muralista mexicano Diego Rivera, el perro le gustó tanto que se dio a la tarea de coleccionar más ejemplares de esta raza hasta el punto de que se convirtió en un criadero. Por su parte el directivo del equipo se empeñó en conseguir un ejemplar rojo, este color es muy codiciado para los amantes de esta raza ya que solo uno de cada cien ejemplares nace con esta tonalidad.

En el criadero familiar nació un perro con esta nacionalidad, el cual Jorge Hank adoptó como su perro personal y le dio el nombre de El Hermoso, ejemplar que ganó una gran cantidad de concursos en varios países. Al morir y por el gran cariño que le tenía, el directivo quiso hacer un homenaje, el perro fue disecado y puesto en la hacienda. El encargado oficial del criadero, Ricardo Fiorasteri, mandó hacer un logo que fuera la imagen que representará a dicho criadero, pero no fue utilizada. Tiempo después, cuando Hank adquiere la franquicia del club Tijuana decidió darle la imagen que antes había sido destinada a su criadero, fue así como El Hermoso quedó plasmado en el emblema de Xolos de Tijuana. La estrella que se encuentra en el escudo fue añadida en el año 2012 tras obtener su primer campeonato (Club Tijuana Xoloitzcuintles de Caliente, 2019).

En el 2016 el diario británico Daily Mail lanzó una lista de los 20 escudos de fútbol más bellos del mundo, entre los cuales se encontraba el escudo de los Xolos en el número catorce, compitiendo con escudos de equipos como Liverpool y Roma (Medio Tiempo 2019).

Se puede destacar que El Hermoso no sólo está presente en el uniforme de Xolos, ya que el dueño del equipo es también propietario del casino Caliente el cual es patrocinador de varios equipos de fútbol mexicano y en el signo identificador de éste se encuentra una representación abstracta del Xoloitzcuintle tan importante para la institución.

\section{Análisis de los 14 parámetros de alto rendimiento en los identificadores de los cinco Clubes de fútbol mexicanos}

A continuación, se presenta el análisis realizado a los cinco clubes de fútbol mexicanos, en donde se evidencia cuáles son con los que sí cumplen y con los que no, de acuerdo a su representatividad gráfica. 


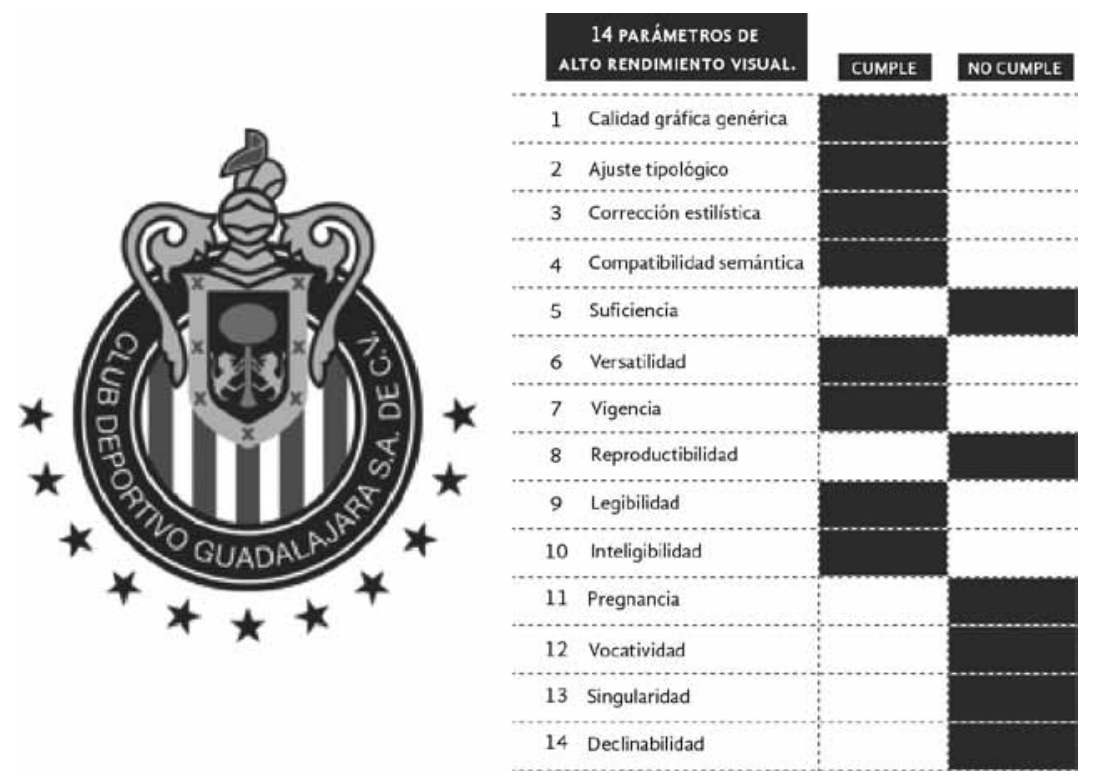

Tabla 4. Análisis del escudo del Club Deportivo Guadalajara. Fuente: Tabla de creación propia; escudo de mejoress (2019, s/p).

En el escudo de la escuadra rojiblanca se pueden identificar que los catorce parámetros de alto rendimiento visual se encuentran divididos, ya que no tiene mucha pregnancia por tener demasiados elementos visuales que imposibilitan que el signo identificador sea recordado con detalles por el usuario, además de que dificultan la reproductibilidad; con respecto a la suficiencia el identificador cuenta con más de los elementos necesarios para lograr comunicar un mensaje claro y adecuado con respecto a la institución, lo que ha generado lograr un excelente posicionamiento tanto dentro como fuera de la perla tapatía, convirtiendo al equipo en uno de los más populares del país.

Los parámetros que se cumplen son los necesarios para que el identificador esté completamente posicionado en el consiente colectivo de la afición mexicana, la calidad gráfica genérica es un elemento claramente presente que hace que sea congruente con la imagen que el equipo tiene ante los usuarios, es importante destacar que el signo identificador no cuenta con todos los parámetros y aun así es capaz de traducir los atributos de la identidad así como se ha generado una serie de elementos gráficos y conductuales que hacen al equipo uno de los principales representantes del fútbol mexicano.

Un aspecto negativo es la singularidad ya que al ser un escudo de armas el signo identificador puede confundirse de categoría y las personas que no estén familiarizados con él, lo pueden atribuir a otra categoría de empresa o asociación. 


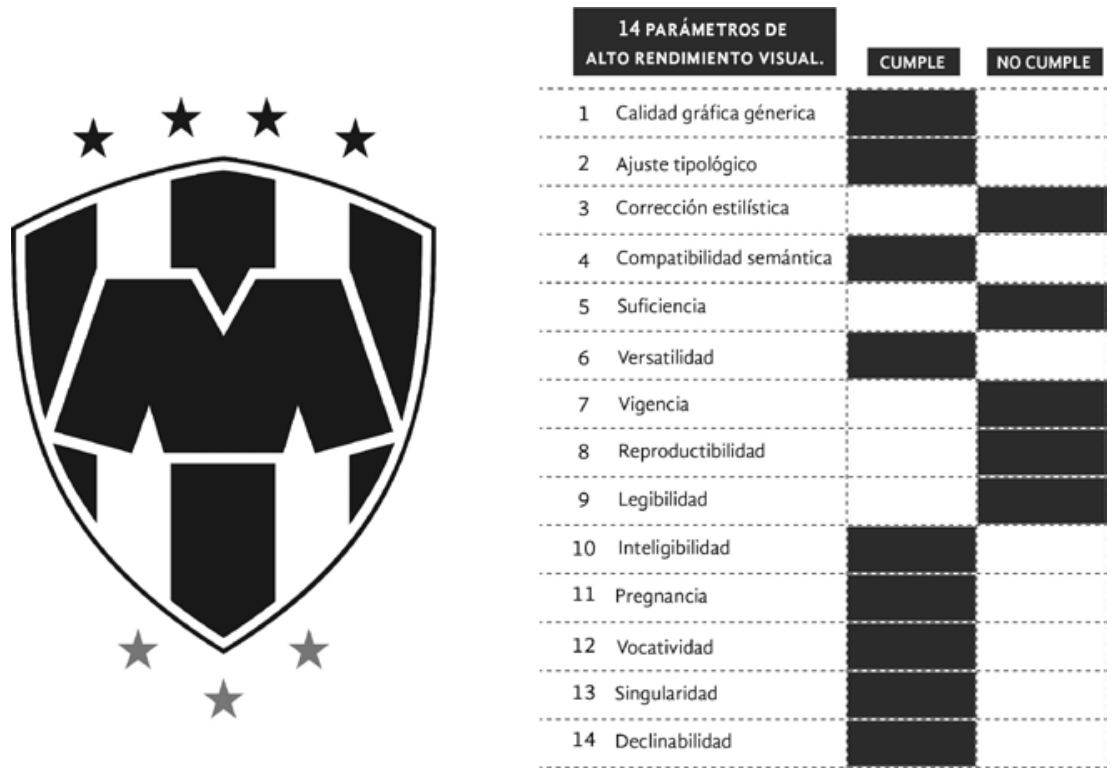

Tabla 5. Análisis del escudo del Club Deportivo Monterrey. Fuente: Tabla de creación propia; escudo de Preciado (2018, s/p).

Escudo en el cual se integran las franjas representativas del uniforme, este elemento lo dota de calidad gráfica genérica, la simplicidad de los elementos lo dotan de ajuste tipológico, los que hace posible que los elementos sean utilizados de manera aislada con el objetivo de generar piezas en las cuales pueden ser aplicados dotándolas de la identidad del equipo sin necesidad de la presencia del escudo. Es importante destacar que se cuenta con compatibilidad semántica ya que el código estilístico en el cual está inserto el identificador es claro, sin necesidad de desambiguar a partir de un elemento lingüístico, como se puede observar es importante que se trabaje en los parámetros que no son cubiertos ya que pueden obstaculizar el crecimiento en el posicionamiento de la institución en la afición mexicana.

La pregnancia es un punto importante que debe ser cubierto para lograr los objetivos de crecimiento; en cuanto a la vocatividad es importante que se genere un identificador que sea capaz de lograrla ya que en el giro en el que se encuentra comparte espacios publicitarios con otros equipos lo que hace que exista una mayor competencia. La singularidad no está presente, lo que puede ocasionar confusiones con respecto a otras escuadras tanto nacionales como internacionales. Es imprescindible buscar que la identificación sea clara con el objetivo de aumentar la pregnancia del signo identificador del equipo. 
La tipografía utilizada en el escudo es la representación de una letra $M$, la cual tiene un tratamiento gráfico que le proporciona las características necesarias para ser identificadas como un signo distintivo de la agrupación, tiene connotación cultural y social que le da sentido al contexto en el que fue generado el identificador, dotándolo de compatibilidad semántica y logrando que los habitantes de la sultana del norte logren adquirir un sentido de pertenencia con el equipo.
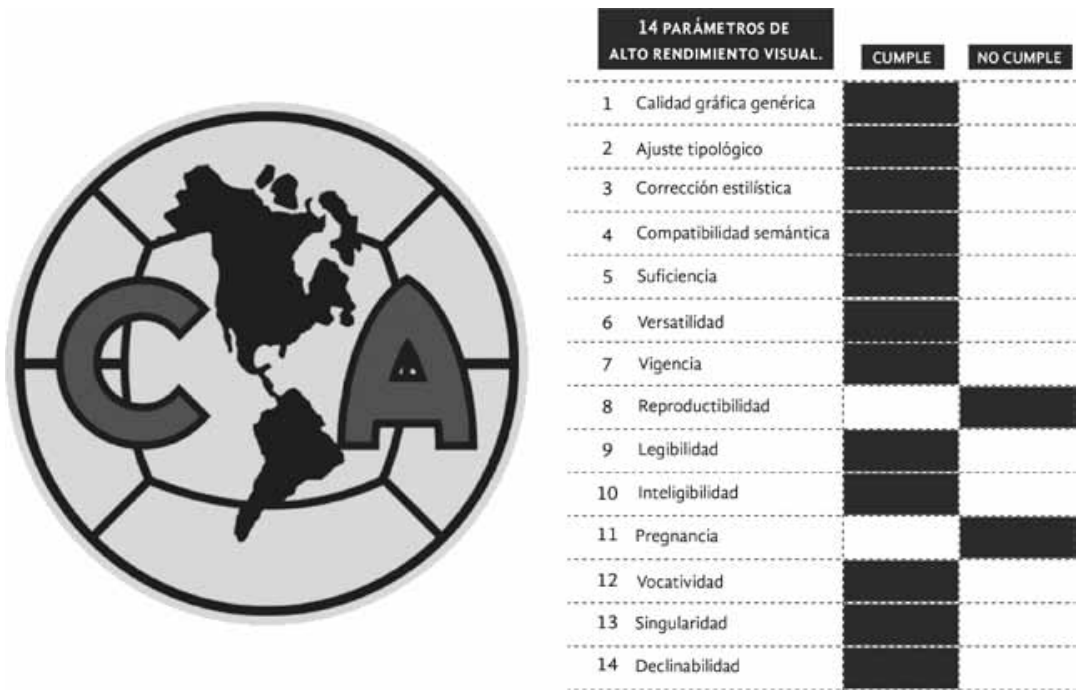

Tabla 6. Análisis del escudo del Club Deportivo América. Fuente: Tabla de creación propia; escudo de Club de Futbol América (2019, s/p).

El escudo del club contiene elementos gráficos que hacen referencia a la disciplina a la que se dedica la institución lo que enfatiza que cuenta con el parámetro de declinabilidad, este parámetro lo dota de las características necesarias para ser comprendido de manera óptima en función a la actividad que se realiza en el club, no existe un signo identificador en el fútbol nacional o internacional que tenga características similares, lo que incrementa la singularidad y lo convierte en una pieza única, esto es un punto que hace la afición se sienta aún más conectado con la institución ya que no hay en el mundo otro equipo igual. El balón de fútbol posiciona al escudo en la categoría en la cual está inserto es decir que al observar es evidente que se trata de un equipo de fútbol. 
Un parámetro de suma importancia tanto para la funcionalidad del signo como para el crecimiento económico del mismo es la reproducibilidad, ya que al facilitar la reproducción se favorece la disminución de los costos de producción, los cuales se pueden invertir en activos para la institución.

La gama cromática utilizada en el diseño corresponde a los históricos colores azul-crema, los cuales desde los inicios del club deportivo han sido un elemento de identificación que se ha posicionado en la afición y el cual ha logrado generar un sentido de pertenencia, logrando que la vigencia del elemento gráfico sea un elemento que ha logrado que el equipo crezca de manera sustancial al percibirlo como un equipo de tradición en México también se incluye el rojo, el cual genera un acento de color y contraste que incrementa la vocatividad logrando la atención de las personas que están en contacto con él, además de que cuando comparte espacios publicitarios con otras escuadras es uno de los más llamativos logrando así el objetivo de comunicación principal.

Con respecto a la tipografía se puede observar que es palo seco, con rasgos orgánicos, además presenta una estructura modular que ha sido modificada para mejorar su reproductibilidad, el cual es un parámetro indispensable para el crecimiento económico e identificatorio del equipo, ya que al lograr una unificación que cuente con la reproducibilidad adecuada, los gastos de producción disminuirán en forma significativa, además ayuda a lograr una mayor pregnancia en el consciente colectivo de la afición. Es importante que la representación del continente se trabaje para lograr una mejor sintetización ya que toda la reproductibilidad ganada con la tipografía se pierde al contar con una forma tan compleja que hace más complicada la reproducción, echando abajo el parámetro de la reproductibilidad factor que incide en el gasto de la institución.

Estamos hablando de un identificador con un gran número de parámetros impactados, lo que le da una calidad mayor a la de muchos equipos de fútbol, aunque es importante que se trabaje con la pregnancia ya que todos los parámetros pueden no ser lo suficientemente importantes si no se cumple el objetivo de permanecer en la mente del usuario una vez que deje de tener contacto con el identificador. 

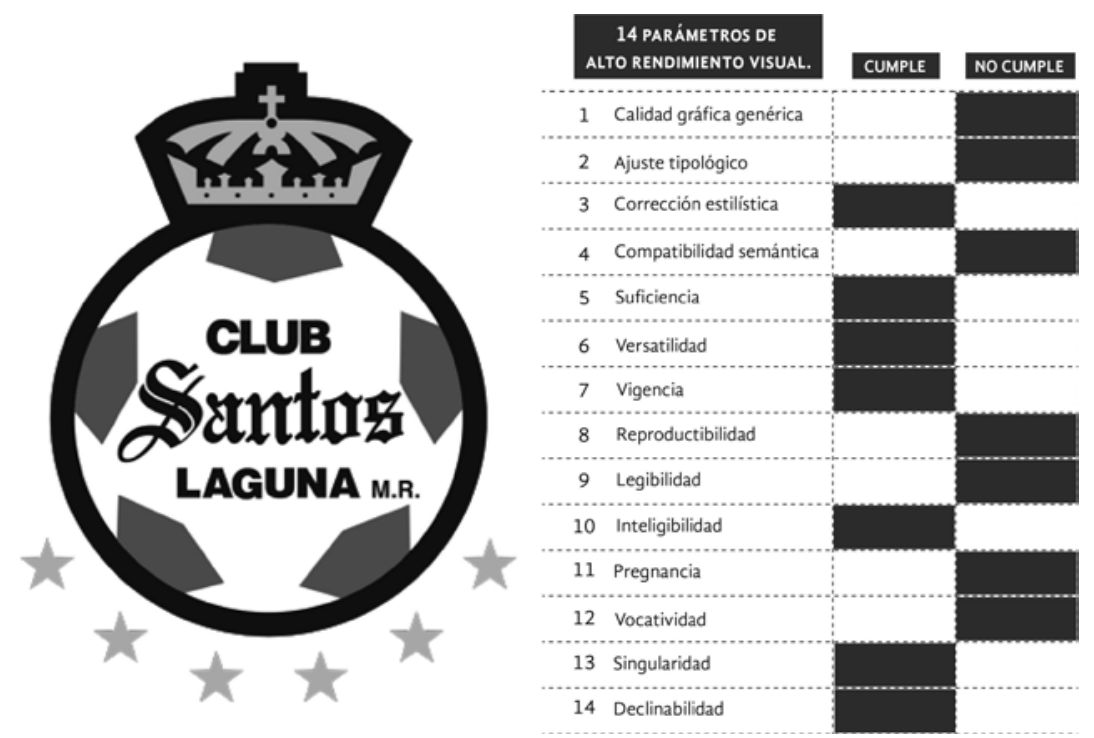

Tabla 7. Análisis del escudo del Club Deportivo Santos Laguna. Fuente: Tabla de creación propia; escudo de Club Santos Laguna (2019b, s/p).

El signo identificador del club Santos Laguna está representado por la síntesis figurativa no realista de un balón de fútbol soccer, el cual dentro contiene los elementos gráficos necesarios para dar el detalle que lo hace identificarse como tal lo cual enfatiza el parámetro de inteligibilidad, que logra que las personas que no están familiarizadas con el equipo tengan una clara idea de lo que es, situación que puede lograr despertar el interés en los posibles aficionados y acercarlos de forma inmediata a él.

La paleta de color utilizada está compuesta por cuatro colores, de los cuales el verde es el que logra mayor identificación entre los aficionados laguneros, lo que dota al gráfico de compatibilidad semántica e incrementa la identificación de la población lagunera con la institución. Puede ser un factor para que sea una de las regiones con mayor afición a su equipo local, ya que al ser destacado como el color institucional, el amarillo logra generar un contraste además de un acento de color que lo hace más llamativo e impactante para el usuario logrando que la vocatividad del signo identificador se incremente ganando protagonismo en cualquier espacio en el que sea colocado.

Este escudo presenta carencias en cuanto a la integración de los parámetros de alto rendimiento que son significativas y le restan calidad al mismo, desde la calidad gráfica genérica al ser un gráfico repleto de elementos incoherentes, hasta tipografías diversas e incluso trazos de poca calidad. Partiendo de esto, se desencadena un ajuste tipológico inexisten- 
te, no es posible lograr una congruencia entre los signos distintivos y el identificador, la compatibilidad semántica es limitada ya que, a pesar de contar con la forma de un balón de fútbol, también se integra una corona la cual hace referencia a la empresa cervecera que comparte dueño con el club deportivo. No tiene pregnancia y es casi imposible recordar cada uno de los detalles que componen el signo identificador y es posible incluso que sea retirado de la memoria una vez que no se tenga contacto con él, además de que puede ser confundido por su estructura general ya que cuenta con rasgos similares a otros identificadores lo que deja a la singularidad fuera del panorama, lo que es otro elemento para que el posicionamiento sea difícil de lograr fuera del contexto en el que el equipo fue creado.
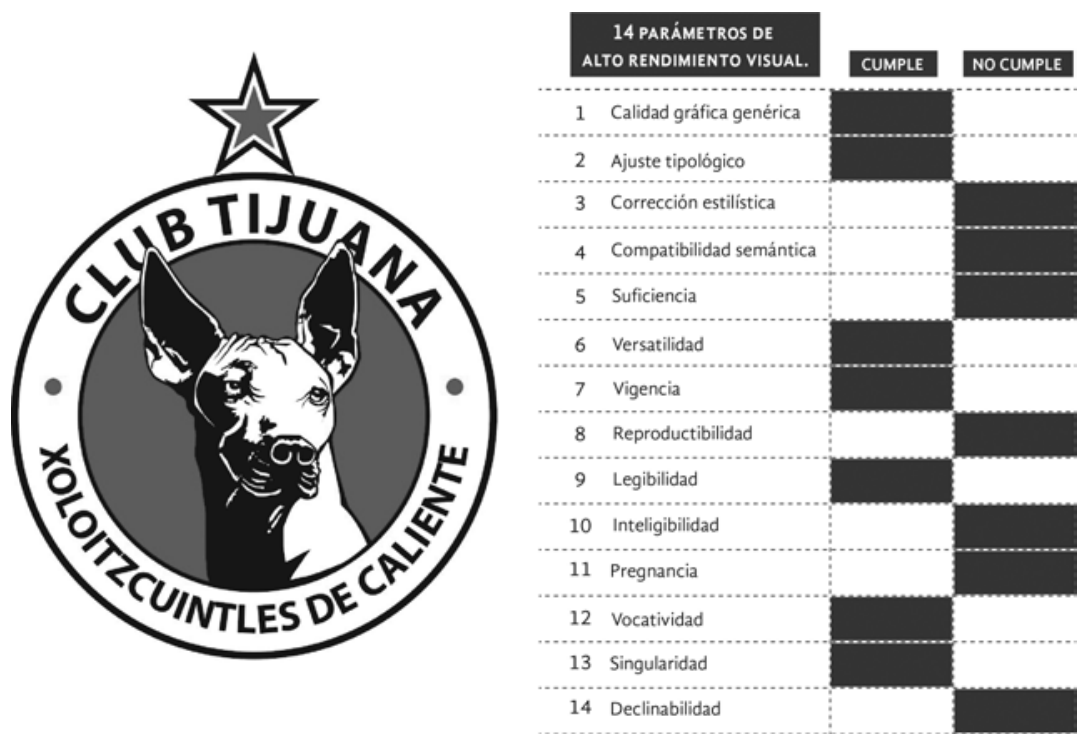

Tabla 8. Análisis del escudo del Club Deportivo Tijuana. Fuente: Tabla de creación propia; escudo de Liga MX (2019b, s/p.).

En el caso de los Xolos de Tijuana se tienen elementos fuera de lo convencional, la estructura es circular, que dentro de los códigos estilísticos principales de los escudos de fútbol hace que se logre de manera parcial la declinabilidad, pero el elemento icónico principal sale completamente de este parámetro ya se trata de la representación figurativa no realista de un perro asociado a las culturas prehispánicas mexicanas lo cual genera confusión en 
el aficionado que no está relacionado con el equipo al poder interpretar el identificador como perteneciente a otra categoría. Otro parámetro que es inexistente en este escudo es la reproducibilidad ya que la cantidad de detalles es considerable, lo que hace que no sea apto para cualquier soporte o sistema de impresión.

Se puede observar como la tipografía sigue un patrón de simplicidad y fácil lectura, lo que ayuda a simplificar y no generar mayor detalle o que se produzca una competencia con el elemento dominante que en este caso es la síntesis del can. Ambos elementos tienen un alto índice de inteligibilidad lo cual permite que se identifiquen claramente los elementos que componen al escudo, en cambio la legibilidad se ve comprometida ya que no es posible que los elementos sean percibidos con claridad en condiciones en las cuales se presente el escudo en un tamaño menor a lo utilizado convencionalmente.

La singularidad es un parámetro que cumple a la perfección el escudo, ya que ningún otro equipo de fútbol nacional o internacional tiene las características que este tiene, lo que enfatiza e incrementa el sentido de pertenencia que ha logrado tanto con los habitantes de Tijuana como en la República Mexicana al presentar como elemento principal a un animal cien por ciento nativo de esta región.

A pesar de ser considerado uno de los escudos más bonitos del mundo, hace falta pensar en los elementos técnicos y de diseño que pueden potencializar el impacto estético y conceptual del elemento gráfico, es posible que el escudo tenga mejoras en cuanto a la pertenencia, suficiencia y compatibilidad semántica de manera ajustando el estilo para lograr impactar a los parámetros de alto rendimiento de manera que además de hermoso sea funcional tanto en la parte de comunicación como en el aspecto técnico cumpliendo impactando así todas las dimensiones que debe contener un buen diseño.

\section{Conclusiones}

Los equipos más representativos del fútbol mexicano están apostando por la mejora de la identidad gráfica, del análisis de los 14 parámetros de alto rendimiento con se concluye en los identificadores de los equipos algunos puntos que pueden tomarse como referencia en la creación y manejo de un identificador gráfico y esto es en primer instancia, que deben cumplir con los parámetros mínimos de su calidad gráfica genérica para hacerlo congruente con la imagen que el equipo tiene ante los aficionados.

Ahora bien, la simplicidad de los elementos los puede dotar de ajuste tipológico, ya que los elementos del escudo, al ser usados de manera aislada lo dotan de la identidad del equipo sin necesidad de la presencia del escudo como una alternativa gráfica. Los elementos que enfatizan el parámetro de declinabilidad y simplicidad lo convierte en un identificador único su afición; por otro lado, la inteligibilidad puede lograr cuando las personas que no están familiarizadas con el equipo tengan una idea clara de lo que trata de expresar el deporte, $y$, por último, utilizar el parámetro de singularidad provoca sentido de pertenencia. Es importante señalar que en la mayoría de los escudos las gamas cromáticas utilizadas en el diseño hacen referencia a los históricos colores de cada equipo. 
Esta identidad, que para aquellos que gustan de este interesante deporte, aficionado o no, requiere de elementos como los cánticos de lucha, la tradición del equipo, pero sobre todo los colores, los gráficos, que representa a un equipo de fútbol traducido en un escudo deportivo, que para muchos es el estandarte que defiende su campo, su tierra, su orgullo, equiparable a aquellos estandartes que alentaban en las batallas por la supervivencia y la defensa de una patria, ahora son usados para alentar la defensa de una cancha.

Cuando estos gráficos son observados, aquellos que sean ajenos a su identidad, lograrán encontrar una plática histórica de un pueblo deportivo que busca contar su pasado en unos trazos y colores que van evolucionando conforme pasa su vida como institución, como equipo, como identidad de un grupo de personas afines a uno de los deportes más rentables y más emocionantes de la historia en nuestro país. Una economía que mueve millones de dólares, en publicidad y marcas, donde su identidad gráfica toma un valor trascendental en la economía de su región, de su país, y en algunos casos a nivel global. Este análisis encuentra esos puntos clave en la creación de una identidad mínimos necesarios para impactar un mercado meta para el cual está pensado en llevar el nuevo gráfico y lograr un impacto, ejemplificado a través del análisis de los escudos de estos cinco equipos de fútbol mexicano.

\section{Referencias Bibliográficas}

América retail. (2018, 7 de julio): México: la pasión del fútbol en los hogares mexicanos. Obtenido de https://www.america-retail.com/mexico/mexico-la-pasion-del-futbol-enlos-hogares-mexicanos/

Asociación de coleccionistas de Jerseys del Guadalajara (2018, 2 de marzo): La evolución del escudo del Club Deportivo Guadalajara primera parte [Twitter] Obtenido de https:// mobile.twitter.com/_acjg_/status/969600969913794561

Chaves, N. y Belluccia, R. (2008). La marca corporativa. Gestión y diseño de símbolos y logotipos. Argentina: Paidós

Club de Futbol América (2019): Época. Obtenido de https://www.clubamerica.com.mx/ historia/

Club de Futbol Monterrey Rayados. (2019). Historia. Obtenido de https://www.rayados. com/monterrey/historia

Club Santos Laguna (2019a): Historia. Obtenido de https://www.clubsantos.mx/es/conte nidos/club_historia

Club Santos Laguna (2019b): Escudo. Obtenido de https://www.clubsantos.mx/es/conte nidos/escudo

Club Tijuana Xoloitzcuintles de Caliente (2019): Historia. Obtenido de http://www.xolos. com.mx/historia.html

Cortina, J.M. (2007): Identidad, identificación, imagen. México: Fondo de cultura económica. Del Val, J. (2004): México, identidad y nación. México: UNAM.

ESPN (2017, 29 de julio): Los deportes más populares en México son... obtenido de https:// www.espn.com.mx/otros-deportes/nota/_/id/3424474/los-deportes-mas-populares-enmexico\%C2\%A0son 
Fuentevilla, N. (2009, 5 de mayo): Análisis de identidad y marca de equipos de fútbol: Rayados de Monterrey, un diseño muy rayado. obtenido de https://ideasfrescas.wordpress.com/ author/nandofuentevilla/page/64/

Galván, G. (2019). \#Diseño: La evolución del escudo del Club América. Obtenido de https:// www.dinamo.mx/a113/2015/diseno-la-evolucion-del-escudo-del-club-america

Huerta, J. (2018, 18 de junio): México desconocido. Obtenido de https://www.mexicodescono cido.com.mx/historia-del-futbol-en-mexico-todo-empezo-con-el-juego-de-pelota.html

Ideas Frescas (2012, diciembre 6): ¡Muy interesante! Análisis del logo de las Chivas de Guadalajara. Obtenido de http://ideasfrescas.com.mx/muy-interesante-analisis-del-logode-las-chivas-de-guadalajara/

Liga MX (2019a): Club de Fútbol Monterrey. Obtenido de https://liga-mx.fandom.com/es/ wiki/Club_de_Fútbol_Monterrey

Liga MX (2018): Club santos se toma la foto oficial del AP18. Obtenido de https://ligamx. net/cancha/detallenoticia/25953/club-santos-se-toma-la-foto-oficial-del-ap18

Liga MX (2019b): Club Tijuana. Obtenido de https://ligamx.net/cancha/club/5/tijuana

Medio Tiempo (2019) Escudo de Xolos fue elegido entre los más bellos del mundo. Obtenido de https://www.mediotiempo.com/futbol/liga-mx/escudo-de-xolos-fue-elegido-entrelos-mas-bellos-del-mundo

Mejoress (2019, 19 de enero): Kits Chivas Guadalajara Dream League Soccer 2019-DLS. Obtenido de https://www.mejoress.com/kits-chivas-guadalajara-dream-league-soccer-2019/

Milenio (2019): Así fueron los primeros escudos de equipos en liga. Obtenido de https://www. milenio.com/deportes/futbol/liga-mx-asi-fueron-los-primeros-escudos-de-equipos

Pérez, I. (2018, 26 de julio): Forbes México. Obtenido de https://www.forbes.com.mx/50equipos-de-futbol-mas-valiosos-de-america-en-2018/

Preciado, S. (2018, 27 de septiembre): Barcelona renovó su escudo como otros equipos. Obtenido de https://naciondeportes.com/barcelona-renovo-su-escudo/

Rendón, A. (2019): Los 10 equipos del fútbol mexicano que más afición llevaron a sus estadios en la última década. Obtenido de https://www.90min.com/es/posts/6306725-los-10-equiposdel-futbol-mexicano-que-mas-aficion-llevaron-a-sus-estadios-en-la-ultima-decada

Revista Mira. (2018): La historia del fútbol en México. Obtenido de https://www.revistamira. com.mx/2015/11/14/la-historia-del-futbol-en-mexico/

Zamora, B. (2018): 75 años de historia de futbol profesional en México. Obtenido de https:// www.futboltotal.com.mx/especial-ft/75-anos-de-historia-del-futbol-profesionalmexico/2018/10

Abstract: In Mexico, soccer becomes a passion for many fans, and the culture surrounding this sport is represented by the expression of batons, attendance at stadiums and social gatherings with family and friends. It is the graphic identifiers of the teams and their identity in the shirts that project the essence of each team, causing a strong identification of the fans with their team, fostering a sense of intense belonging to them. This is a phenomenon that few companies can achieve so reliably in the mind of the consumer and 
even get to awaken an indescribable love. This document documents the evolution of the graphic identifiers of the five most representative teams of Mexican soccer according to the magazine Forbes, Mexico 2018 that include Guadalajara, Monterrey, América, Santos Laguna, and Tijuana, and some of the effects that they produce in their followers.

Keywords: Identity - Graphic identifiers - Soccer - Mexican culture - Sense of belonging.

Resumo: No México, o futebol se torna uma paixão para muitos fãs, e a cultura em torno deste esporte é representada pela expressão de cassetetes, participação em estádios e reuniões sociais com familiares e amigos. São os identificadores gráficos das equipes e sua identidade nas camisetas que projetam a essência de cada equipe, provocando uma forte identificação dos torcedores com sua equipe, fomentando uma sensação de intenso pertencimento a eles. Esse é um fenômeno que poucas empresas conseguem alcançar de maneira tão confiável na mente do consumidor e até conseguem despertar um amor indescritível. Este documento documenta a evolução dos identificadores gráficos das cinco equipes mais representativas do futebol mexicano, de acordo com a revista Forbes, México 2018, que inclui Guadalajara, Monterrey, América, Santos Laguna e Tijuana, e alguns dos efeitos que eles produzem em seus seguidores.

Palavras chave: Identidade - Identificadores gráficos - futebol - Cultura mexicana - Senso de pertencer.

[Las traducciones de los abstracts fueron supervisadas por el autor de cada artículo] 